\title{
Conservative Management and Planned Surgery for Periviable Advanced Extrauterine Abdominal Pregnancy with Favorable Outcome: Report of Two Cases
}

\author{
Hassan M. Harirah, MD ${ }^{1} \quad$ J. Michael Smith ${ }^{2} \quad$ C. Luke Dixon, MD ${ }^{1}$ Gary D. V. Hankins, MD ${ }^{1}$ \\ ${ }^{1}$ Division of Maternal Fetal Medicine, Department of Obstetrics and \\ Gynecology, The University of Texas Medical Branch at Galveston, \\ Galveston, Texas \\ 2 The University of Texas Medical Branch at Galveston,

\begin{abstract}
Address for correspondence Hassan M. Harirah, MD, Division of Maternal Fetal Medicine, Department of Obstetrics and Gynecology, The University of Texas Medical Branch, 301 University Boulevard, Galveston, TX 77555-0587 (e-mail: hmharira@utmb.edu).
\end{abstract} Galveston, Texas

Am J Perinatol Rep 2016;6:e301-e308.

\author{
Abstract \\ Keywords \\ - advanced abdominal \\ pregnancy \\ - extrauterine \\ - periviable \\ - obstetric ultrasound \\ - oligohydramnios \\ - MRI \\ - placenta \\ - conservative \\ management
}

\begin{abstract}
Advanced abdominal pregnancy is an extremely rare condition that poses diagnostic and management challenges. A high index of suspicion and careful assessment of the patient's symptoms, supplemented with obstetric ultrasound, and magnetic resonance imaging, are crucial for timely diagnosis and management to prevent life-threatening complications. The presence of periviable fetuses in advanced abdominal pregnancies increases the challenge to achieve a balance between maternal and fetal benefits and risks. Early diagnosis and management decisions via a multidisciplinary approach and planned delivery are of paramount importance to minimize complications and achieve favorable maternal and fetal outcomes. Even in the setting of oligohydramnios and suspected preterm premature rupture of membranes, in-patient conservative management and an individualized planned surgical approach that includes removing or leaving the placenta in place are appropriate for managing the periviable abdominal pregnancy.
\end{abstract}

Abdominal pregnancy is a rare form of extrauterine gestation that accounts for approximately $1.4 \%$ of all ectopic pregnancies and often leads to poor maternal and fetal outcomes., ${ }^{1,2}$ The maternal mortality rate associated with abdominal pregnancies is almost eight times greater than that of tubal pregnancies and 90 times greater than that of normal intrauterine pregnancies. $^{3}$

Abdominal pregnancy is a form of ectopic pregnancy that occurs when the developing blastocyst/embryo implants onto the peritoneum or abdominal organs instead of the endometrium of the uterine cavity. The underlying mechanism by which this aberrant implantation occurs involves either primary implantation or secondary implantation. Primary implantation occurs when the fertilized ovum and developing blastocyst is expelled into the abdominal cavity from the

received

June 20, 2016

accepted after revision

July 14, 2016
DOI http://dx.doi.org/

10.1055/s-0036-1588003. ISSN 2157-6998. fimbriated end of the fallopian tube. Secondary implantation occurs when an embryo that was previously implanted in the fallopian tube is released into the abdominal cavity secondary to tubal abortion or tubal rupture. ${ }^{1}$

Abdominal pregnancies pose unique diagnostic and therapeutic challenges. Patients with abdominal pregnancies can have variable clinical presentations, and the physical examination may be inconclusive, thus delaying diagnosis until an advanced stage of gestation. ${ }^{4}$ Advanced abdominal pregnancy with a viable fetus is an extremely rare condition, which demands a high index of suspicion for its correct diagnosis and skilled surgical management. A high perinatal mortality rate of 45 to $90 \%$ due to catastrophic hemorrhage has been reported in advanced abdominal pregnancies. ${ }^{5,6}$ The fetus survives in only 10 to $20 \%$ of reported cases, and $21 \%$ of babies
Copyright $\odot 2016$ by Thieme Medical Publishers, Inc., 333 Seventh Avenue, New York, NY 10001, USA. Tel: +1(212) 584-4662.
License terms

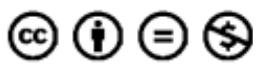


who survive through birth have significant deformities including limb defects, facial and cranial asymmetry, joint abnormalities, and central nervous system malformation due to loss of amniotic fluid. ${ }^{7}$ There is currently no well-established standard of care for the management of advanced abdominal pregnancy, particularly when it is diagnosed in periviable gestational age.

We report two cases of advanced abdominal pregnancies in which the pregnancies progressed to near term and resulted in viable infants and favorable maternal outcomes.

\section{Case 1}

A 34-year-old African American female, gravida 1, para 0, at 28 weeks and 4 days gestation was transferred to our institution from an outside hospital for abdominal pregnancy diagnosed on magnetic resonance imaging (MRI). The patient was asymptomatic and denied abdominal pain, vaginal or rectal bleeding, or abnormal bowel movements. She had a history of infertility and left hydrosalpinx noted on hysterosalpingogram, followed by diagnostic laparoscopy, which showed extensive intra-abdominal adhesive disease requiring adhesiolysis. No uterine anomalies were reported.

On admission, ultrasound examination confirmed an abdominal pregnancy with an appropriately grown fetus $(1,344 \mathrm{~g})$ in a longitudinal lie on the left side of the maternal abdomen with fetal head in the left lower quadrant and decreased amniotic fluid. Repeat MRI with contrast of abdomen and pelvis at our institution confirmed an extrauterine abdominal pregnancy. The fetus was noted to occupy the left side of the abdomen, and a large placental mass occupied much of the right side of the abdomen with the medial placental edge close to the midline. The placenta was attached to the majority of the omentum and small bowel mesentery, closely apposed to the abdominal wall without clear signs of invasion, and abutted the right psoas muscle and the right ureter. The placenta was noted on the right outside wall of the uterus and showed marked contrast enhancement making it indistinguishable from the uterus and right broad ligament (-Fig. 1A-C). No dilated bowel suggesting obstruction was noted. The urinary bladder and colon appeared uninvolved. Amniotic fluid was noted to be decreased, and fetal pulmonary hypoplasia was suspected.

A multidisciplinary conference was assembled including maternal-fetal medicine, anesthesiology, neonatology, and gynecological oncology to discuss her case. Expectant management was suggested to allow for fetal lung development; however, this option is associated with the risk of emergent intervention in the case of fetal distress. The other option is proceeding with delivery to avoid emergency surgery; however, this option is associated with increased risk of fetal risk of pulmonary hypoplasia as it was suggested by the MRI findings and the decreased amniotic fluid. The patient was counseled extensively about these two options, and she opted for delivery. She was counseled regarding delivery via laparotomy, risk of hemorrhage and blood transfusion, and possible damage to her bladder and bowel.

At 29 weeks and 3 days, exploratory laparotomy via a suprapubic left paramedian incision (to avoid going through the placenta) was performed under general anesthesia. Upon entering the peritoneal cavity, the amniotic sac was opened, and a female infant weighing $1,225 \mathrm{~g}$ was delivered in cephalic presentation with Apgar score 6 and 9 at 1 and 5 minutes, respectively. The placenta was noted to be attached to the right broad ligament and the right side of the posterior abdominal wall. The gynecologic oncology team proceeded with the removal of the placenta. Successful removal of approximately $75 \%$ of placental tissue was
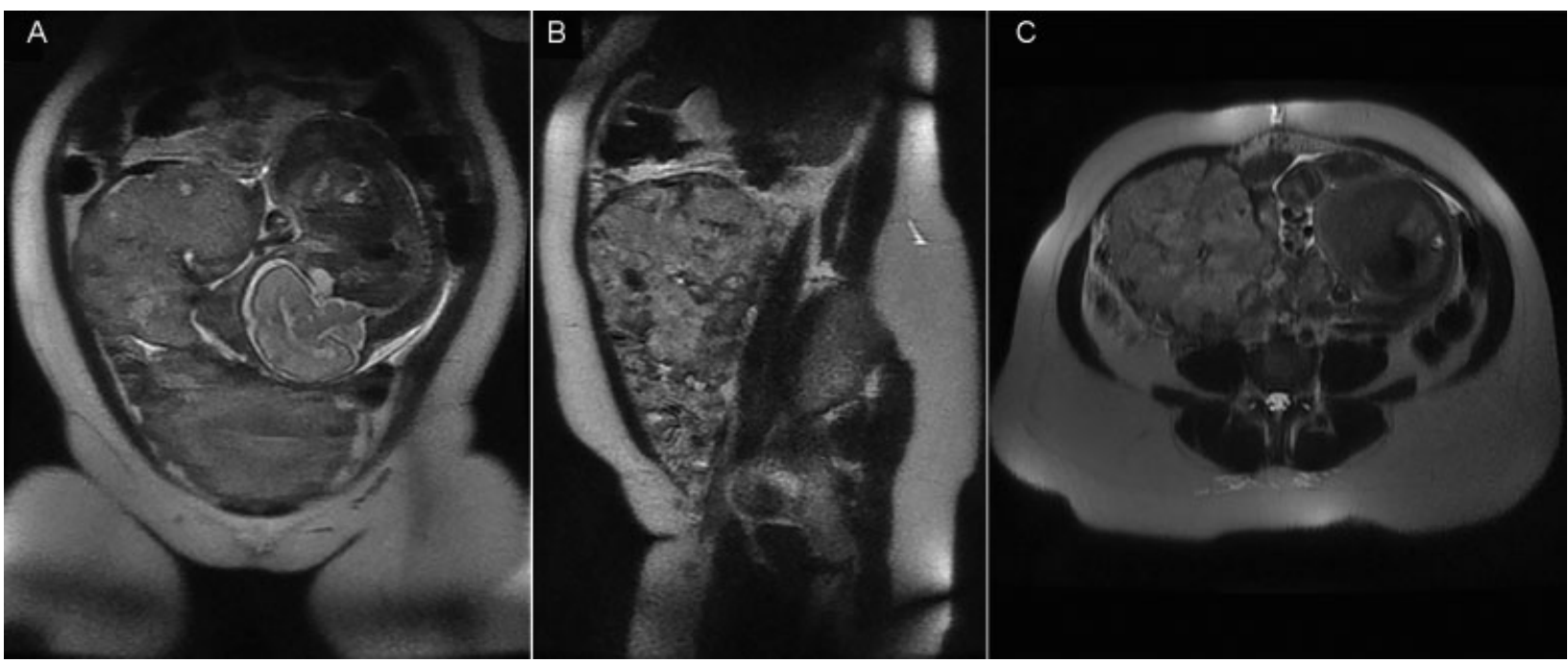

Fig. 1 (A) Coronal view of MRI abdomen and pelvis showing abdominal pregnancy in longitudinal lie and on maternal left hemiabdomen. Placenta is seen on the right side of maternal abdomen juxtaposed to the abdominal wall and above an empty uterus. (B) Right side sagittal view of MRI abdomen and pelvis showing heterogeneous mass of the placenta attached to the omentum and occupying the right side of the maternal abdomen, reaching anteriorly to the anterior abdominal wall. (C) Transverse view of MRI abdomen showing the cross-section of fetal chest and heart on left side of maternal abdomen and placenta on the right side of abdomen. The placenta did not cross the midline. MRI, magnetic resonance imaging. 
achieved, which required omentectomy. No bladder or bowel injury occurred. Intraperitoneal bleeding was encountered during placental removal. The patient received an intraoperative transfusion of five units of packed RBCs, six packs of platelets, and six packs of cryoprecipitate. The patient tolerated the procedure very well and transferred to the surgical intensive care unit for close monitoring. The postoperative course was uncomplicated, and the patient and her neonate were discharged home after 5 days. She is now 7 years remote from the surgery and receiving follow-up by her referring provider with no complications reported.

\section{Case 2}

A 23-year-old African American woman, gravida 7, para 3,215, presented at 23 weeks of gestation to our perinatal ultrasound diagnostic unit for a routine fetal anatomy survey. The ultrasound findings revealed an empty uterus in the maternal left lower abdominal quadrant (-Fig. 2A, B). The endometrium measured $11.4 \mathrm{~mm}$ in thickness with no intrauterine gestation. An echogenic $16.0 \times 10.0 \times 9.2 \mathrm{~cm}$ heterogeneous mass consistent with placenta was noted on the maternal right lower quadrant of the abdomen ( - Fig. 2C, D). An extrauterine intra-abdominal fetus was noted in the maternal upper abdomen in a transverse lie with the fetal head to the maternal left side. No amniotic fluid sac and fluid were noted ( - Fig. 2E). The composite fetal biometry was consistent with 21 weeks and 4 days, and visualization of fetal anatomy was limited by fetal position and lack of amniotic fluid. Free fluid was noted in the maternal cul-de-sac and left adnexa (-Fig. 2F).
Review of the patient's history indicated that she conceived spontaneously and had no prior abdominal surgery. She started her prenatal care at 19 weeks gestation with fundal height consistent with her dates. Her obstetric history included three term deliveries, two preterm deliveries, one elective first-trimester termination, and mild preeclampsia in two of her term pregnancies. Her gynecological history was significance for recurrent episodes of genital chlamydia trachomatis infection for which she received treatment after each episode. A month earlier to her presentation, the patient was seen in labor and delivery triage with a complaint of intermittent abdominal and back pain. Evaluation including abdominal ultrasound examination suggested an intrauterine gestation consistent with her dates, and her symptoms were attributed to constipation. She received azithromycin for a chlamydial infection and flagyl for bacterial vaginosis and was discharged home.

The patient was informed about the findings that were consistent with abdominal pregnancy. She was counseled about the increased risk for intra-abdominal bleeding and the previable gestation of her fetus and was admitted for inpatient management. MRI of the abdomen and pelvis confirmed the findings, and the extrauterine fetus noted in the maternal left hemiabdomen with fetal head in the left upper abdominal quadrant and a very small amount of amniotic fluid surrounding the fetus (-Fig. 3A). The placenta was noted to involve the anterior aspect of the dome of the uterus with suspected involvement of the rectosigmoid junction, sigmoid colon, and right ovary. The placenta was a juxtaposed to the right rectus abdominis muscle, ureters, and bladder without

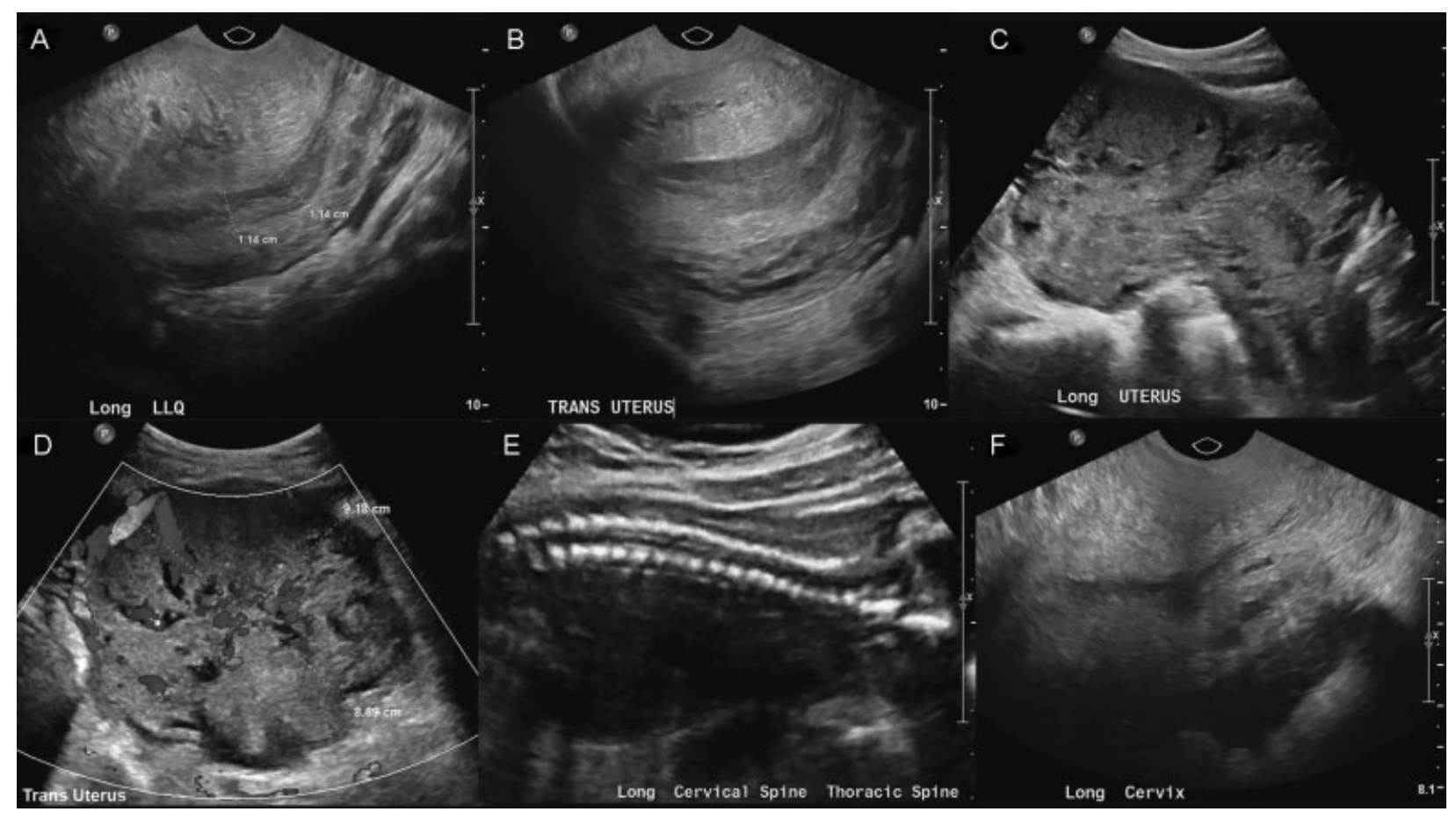

Fig. 2 (A) Longitudinal sagittal view of transvaginal ultrasound showing an empty uterine cavity with endometrial thickness 11.4 mm. (B) Transverse view of transvaginal ultrasound showing an empty uterine cavity and thickened endometrium. (C) Sagittal view of transabdominal ultrasound showing placental tissue on top of the uterine fundus. (D) Transverse view of transabdominal ultrasound showing heterogeneous placental tissue in the abdominal cavity with no uterine wall identified. (E) Transabdominal ultrasound showing an extrauterine intra-abdominal fetus with oligohydramnios. (F) Longitudinal transvaginal ultrasound view showing free fluid in the cul-de-sac posterior to the cervical canal. 


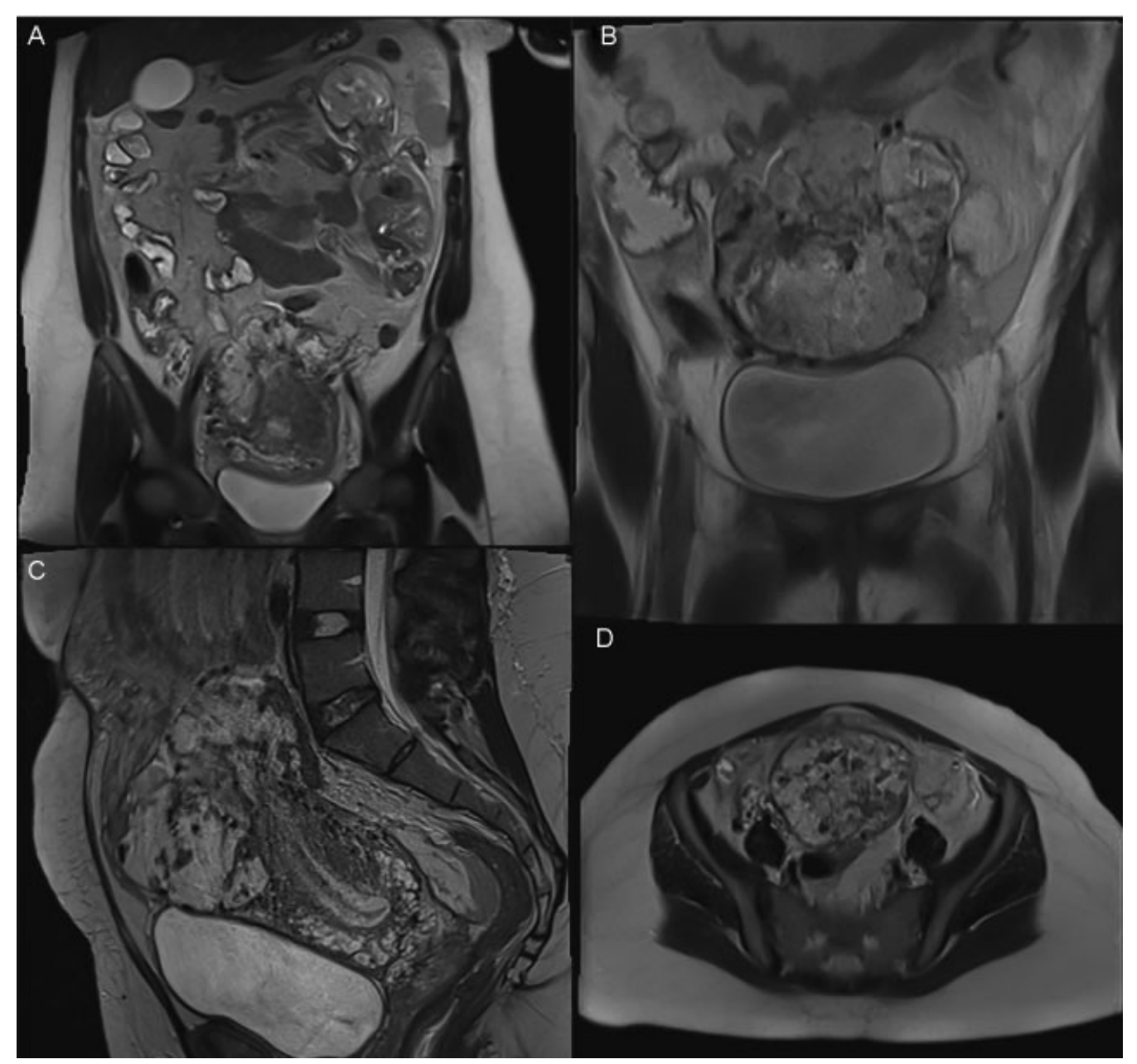

Fig. 3 (A) Coronal view of MRI of posterior part of maternal abdomen showing an extrauterine intra-abdominal fetus with oligohydramnios. (B) Coronal view of MRI of anterior part of maternal abdomen showing a heterogeneous placenta on top of the uterine fundus and bladder. (C) MRI sagittal view of maternal abdomen and pelvis showing the placental location on top of the dome of the empty uterus and juxtaposed to rectus abdominis muscle and bladder. (D) MRI transverse view of maternal pelvis showing placenta in the anterior portion of the pelvis on top and posterior to the uterus, reaching down to the cul-de-sac. MRI, magnetic resonance imaging.

definite involvement ( - Fig. 3B-D). A small amount of free fluid was noted in the abdomen and pelvis.

Laboratory results showed microcytic hypochromic anemia with hematocrit $20.2 \%$ and hemoglobin $6.4 \mathrm{~g} / \mathrm{dL}$. However, she had stable vital signs and was asymptomatic other than mild abdominal pain. The patient's condition of periviable advanced abdominal pregnancy was discussed in a multidisciplinary conference including maternal-fetal medicine, anesthesiology, neonatology, gynecological oncology, and vascular surgery. There was a debate about the increased risk for maternal intra-abdominal hemorrhage and the need to perform a controlled laparotomy to remove the pregnancy before viability and avoid further involvement of the abdominal organs by the growing placenta. However, because her clinical status was stable, the fetus was previable, and significant legal restriction in the state of Texas on the termination of pregnancy, the consensus was for expectant management unless there was clear evidence of intra-abdominal bleeding.

The patient was counseled about the plan, and she indicated that her maternal health was the primary concern. She opted to decline fetal monitoring to avoid an intervention with emer- gency laparotomy for fetal distress. To determine if there was evidence of ongoing intraperitoneal bleeding, the patient was transfused with four units of packed red blood cells (PRBCs) with a plan to check her hematocrit 6 hours after completion of transfusion. Her hematocrit improved to $31.5 \%$ and did not drop on further follow-up, indicating an absence of ongoing intraperitoneal bleeding. Her condition remained stable, and no further drop of hematocrit was observed. Fetal well-being was monitored only with fetal movement and checking fetal heart tones if there was no fetal movement perceived. At 24 weeks' gestation, she received two doses of $12-\mathrm{mg}$ betamethasone 24 hours apart to enhance fetal maturity and magnesium sulfate infusion for 12 hours for neuroprotection.

The patient remained hemodynamically stable and without significant pain for the remainder of the pregnancy. At 27 weeks and 6 days of gestation, a repeat ultrasound for fetal growth revealed fetal growth restriction at the 7 th percentile with fetal head circumference and a biparietal diameter less than the 3rd percentile. Anhydramnios was noted, and umbilical artery Doppler velocimetry revealed a normal S/D ratio. Due to fetal growth restriction, a plan was made 
to repeat an ultrasound for fetal growth in 14 days in addition to fetal movement count monitoring and ultrasound fetal heart tones if there was decreased or absent fetal movement.

At 29 weeks and 6 days of gestation, a follow-up ultrasound for fetal growth and umbilical artery Doppler velocimetry revealed the persistence of fetal growth restriction at less than the 3rd percentile, anhydramnios, and an elevated S/D ratio of 4.3 with diastolic flow present. A repeat MRI of the abdomen and pelvis showed the fetus in the transverse lie in the upper abdomen. The placenta was also noted along the fundus and body of the uterus, extending to the distal sigmoid colon and rectosigmoid junction. The placenta was also noted adjacent to the bladder, right rectus abdominis muscle, and right ovary. Based on findings of persistent growth restriction, anhydramnios, and worsening umbilical artery Doppler parameters, a plan was made for delivery.

On the night before delivery, the patient received a rescue course of betamethasone, an additional magnesium sulfate intravenous infusion for 12 hours for neuroprotection, and a bowel prep. At 30 weeks' gestation, delivery was performed under general anesthesia. Before surgery, the fetal position was delineated by ultrasound. The fetus was noted in a transverse lie in the maternal upper abdomen with fetal head on the right side, and placental upper edges were identified 1 to $2 \mathrm{~cm}$ below the maternal umbilicus. Therefore, laparotomy was performed via a midline supraumbilical incision. A male infant weighing $1,085 \mathrm{~g}$ was delivered in cephalic presentation with Apgar score 6 and 8 at 1 and 5 minutes, respectively. A short umbilical cord was noted. It was cut and tied twice with 0 -vicryl suture, and the placenta was left in the intra-abdominal cavity. A thoracic epidural catheter was placed for postoperative pain control. The postoperative course was uneventful, and the patient was discharged home after 4 days. She had postpartum follow-up visits without complaints and is in good health. Repeat MRI 6 months after delivery showed significant reduction of the residual placenta to approximately one-third of its volume before delivery (-Figs. 4A-C).
Aside from complications of prematurity, the newborn had a congenital anomaly of the toes of the right foot as the result of an amniotic band. Due to the diagnosis of anhydramnios and suspected preterm premature rupture of membranes (PPROM) at the time of admission, pulmonary hypoplasia was an antenatal concern; however, the neonate was intubated for only the first day of life. The only diagnosis of pulmonary morbidity made during the neonate's initial hospitalization was respiratory distress syndrome. The newborn remained in the neonatal intensive care unit for 26 days and discharged home in stable and after achieving appropriate weight gain.

\section{Discussion}

Advanced abdominal pregnancy is a rare and intriguing condition of ectopic pregnancy, which many obstetricians may not encounter in their practices. The predisposing factors associated with this condition include pelvic inflammatory disease, endometriosis, history of infertility, previous ectopic pregnancy, and delay in initial prenatal care and obtaining an early ultrasound. ${ }^{3,8}$ A history of infertility with an adhesive disease requiring laparoscopic adhesiolysis in case 1 and recurrent chlamydia infection in case 2 was noted. In both cases, there was a delay in starting prenatal care and obtaining an early ultrasound to document the location and gestational age of the pregnancy. In case 2, the patient reported an interesting history of severe abdominal pain after sexual intercourse early in the first trimester, raising the suspicion of tubal abortion or rupture of tubal pregnancy. She reported being seen in the emergency room for this pain and was discharged home. A high index of suspicion is therefore warranted in any patient who is at risk for ectopic gestation experiencing unexplained abdominal pain.

Dramatic presentations of obstetric emergencies have been reported with abdominal pregnancies: hemodynamic instability due to intra-abdominal hemorrhage, fetal demise, or extrauterine pregnancy as an unexpected finding during cesarean delivery after failed induction of labor. These reports

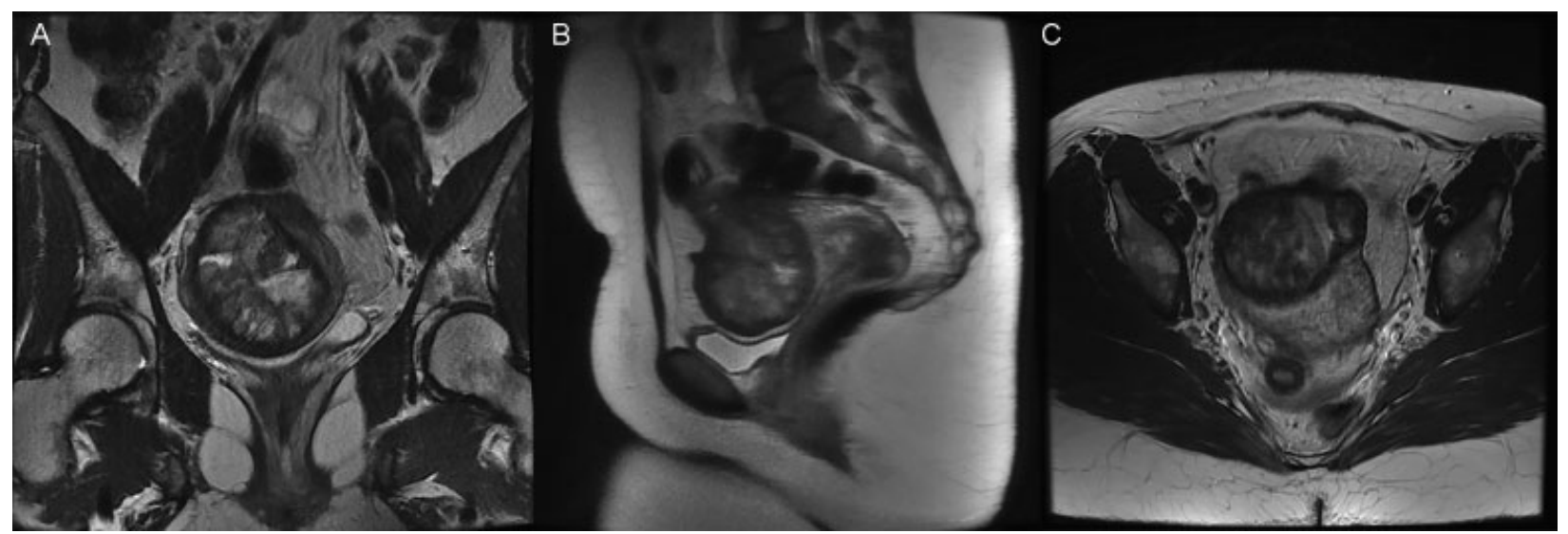

Fig. 4 (A) Six months postdelivery, MRI coronal view of maternal pelvis showing significant reduction of the residual placenta. (B) Six months postdelivery, MRI sagittal view of maternal pelvis showing significant reduction of the residual placenta compared with placental size in - Fig. $\mathbf{3 B}$. (C) Six months postdelivery, MRI transverse view of maternal pelvis showing significant reduction of the residual placenta compared with placental size in -Fig. 3C. MRI, magnetic resonance imaging. 
have originated mainly from developing countries with low imaging resources or access to obstetric care. ${ }^{9}$ Also, extremely rare cases of heterotopic advanced abdominal twin pregnancy with the other twin in utero ${ }^{10}$ and an advanced abdominal twins pregnancy with an empty uterus have been reported. ${ }^{11}$

Diagnosis and management of advanced abdominal pregnancy are still a challenge. Vigilance and careful assessment of the patient's symptoms supplemented with imaging studies are crucial for the timely diagnosis of this rare obstetric occurrence and preventing life-threatening complications. The symptoms of advanced abdominal pregnancies are usually vague and nonspecific, such as abdominal pain or discomfort, vaginal bleeding, decreased/absent or painful fetal movement, and some women are asymptomatic with the condition remaining undiagnosed, given the generous capacity of the abdominal cavity. ${ }^{6,12,13}$

In both cases discussed, the symptoms were a nonspecific trivial abdominal pain, and the diagnosis was missed until an advanced stage of gestation. Findings that might suggest intra-abdominal pregnancy include anemia, abdominal tenderness on palpation, easily palpated fetal parts, abnormal fetal lie, a displaced cervix, and elevated levels of maternal serum alpha-fetoprotein (MSAFP). However, these signs are easily missed due to the rarity of the condition and the low level of suspicion for advanced abdominal pregnancy. ${ }^{14}$ In case 2, an intrauterine pregnancy was reported on her labor and delivery visit a week before her presentation.

Confirmation of the diagnosis of abdominal pregnancy requires imaging studies, mainly via obstetric ultrasound examination. Even before the availability of modern ultrasound, the mainstay diagnostic finding of intra-abdominal pregnancy is the presence of an empty uterus separate from the fetus and an ectopic intra-abdominal placenta. ${ }^{15}$ In a series from the Parkland Hospital, however, only 6 cases of 10 women with advanced abdominal pregnancy were correctly diagnosed by ultrasound. ${ }^{9}$ Therefore, the ultrasound examination must be performed by an experienced ultrasonologist with a high index of suspicion, and confirming that the pregnancy lies within the confines of the uterus is essential with each obstetric ultrasound examination. Other nonspecific ultrasound findings include oligohydramnios, pseudo placenta previa appearance, inability to establish continuity between cervical canal and the amniotic cavity, and the presence of maternal intraperitoneal fluid. ${ }^{5,6,16}$ In addition to diagnosis, determination of the gestational age and potential fetal viability is essential in the management of advanced abdominal pregnancy.

MRI is a useful adjunct tool in confirming the diagnosis and identifying the extent of placental attachment to abdominal organs and vascular structures. ${ }^{9,16}$ Many case reports showed the value of MRI in the preoperative determination of placental attachment to the omentum, ${ }^{17}$ the right lobe of the liver, ${ }^{18}$ and placental vasculature feeding off sacral plexus. ${ }^{19}$ Other reports described shortcomings of MRI: falsely suggesting placental implantation into the cecum which was not confirmed during surgery and failing to localize placental implantation sites compared with better results from extended field-of-view sonography. ${ }^{9,20}$ In our cases, MRI findings added more detailed information about placental location and attachment. In case 1 , the placenta was attached to the omentum and right broad ligament, and this information was helpful in planning omentectomy to remove approximately $75 \%$ of the placental mass. In case 2 , MRI showed the placenta was attached to the rectosigmoid junction and uterine fundus and was juxtaposed to the lower anterior abdominal wall, which was helpful in patient counseling and informed the surgical planning decision of leaving the placenta in place rather than attempting to remove it. On the other hand, MRI in case 1 indicated fetal pulmonary hypoplasia, which was not proven after delivery, and the newborn had no significant breathing problems. Despite limitations associated with imaging studies, we believe it is always wise to perform ultrasound and MRI to gather maximum information about the topography and extent of placental implantation for better surgical planning. In our cases, knowledge of the exact location of each placenta guided our abdominal incisions during laparotomy (left paramedian in case 1 and midline supraumbilical in case 2) to avoid incising through the placenta.

The presence of periviable fetuses in advanced abdominal pregnancies adds to the level of challenge since achieving a balance between maternal and fetal benefits and risks is often a difficult task. While continuation of pregnancies-via expectant management-offers more time for fetal development and maturation, it poses a major risk for spontaneous intraperitoneal bleeding, and unplanned emergent surgical intervention for fetal distress can provoke massive hemorrhage resulting from disruption of the placental vascular bed. Conversely, termination of pregnancy to avoid further placental invasion into maternal organs will increase fetal morbidity and mortality associated with prematurity. There is no consensus about the best way of managing this dilemma. Some have suggested expectant hospitalization of select patients to await fetal maturity. ${ }^{21}$ Others advocate prompt intervention when abdominal pregnancy is diagnosed, as there is a great risk of life-threatening hemorrhage with continuing pregnancy, and a significant portion of these patients will require emergent laparotomy with high maternal and perinatal morbidity and mortality. ${ }^{9}$

In our cases, early diagnosis and management decisions via a multidisciplinary approach and planned delivery significantly minimized complications and achieved favorable maternal and fetal outcomes. The principal objectives were delivery of viable fetuses and careful assessment of placental implantation without provoking hemorrhage. Our approaches for the two cases differed based on careful clinical assessment from the multidisciplinary team and the patients' inputs. Both patients were counseled thoroughly about their options. The patient in case 1 opted for prompt operative intervention, occurring before the introduction of magnesium sulfate protocol for fetal neuroprotection. The gynecologic oncology team in case 1 performed omentectomy and removed $75 \%$ of the placental mass. Intraoperative bleeding was encountered during placental removal, and the patient received a transfusion of blood products. In contrast, the 
patient in case 2 was admitted for inpatient management as long as her clinical status remained stable, and she received magnesium sulfate for neuroprotection before delivery. The patient decided that her safety and health was her primary concern, and she opted against fetal heart monitoring during the expectant management period. Serial ultrasound for follow-up fetal growth and umbilical artery Doppler were implemented in case 2 to monitor fetal well-being when fetal growth restriction was noted. Planned laparotomy and delivery were considered when umbilical artery Doppler velocimetries worsened. In case 2, the placenta was left in place, and no attempt to remove was considered. The operative blood loss was minimal. Both patients tolerated laparotomies well with uneventful postoperative courses. Aside from prematurity complications, both newborns did well in the neonatal intensive care unit and were discharged home healthy. No complication of placental necrosis or peritonitis was noted in either case. Repeat MRI 6 months after surgery showed a significant reduction of the placental volume (-Table 1) in case 2. Similarly, recent developments in management of abnormally adherent placentas showed spontaneous placental resorption occurred in $75 \%$ of cases managed conservatively by leaving placentas in place for an interval of 4 to 60 weeks after delivery. ${ }^{22}$

While conservative management of a periviable pregnancy has been reported, ${ }^{23}$ case 2 is unique for conservative management of an abdominal pregnancy with the suspected diagnosis of PPROM. At the time of her admission, with a normally grown fetus and no amniotic fluid seen by ultrasound, PPROM was presumed. While other studies have reported abdominal pregnancies in the setting of oligohydramnios or anhydramnios, none of them were reported to be managed expectantly. ${ }^{9,24,25}$ Despite the fact that consensus management of PPROM includes antibiotic administration to prolong latency, ${ }^{26}$ we did not opt for this management due to the sterility of the peritoneal cavity. We were, however, concerned with the possibility of severe pulmonary hypoplasia. It is theorized that the lack of amniotic fluid and chronic compression of the fetal lungs in the setting of intrauterine PPROM results in severe fetal pulmonary hypoplasia. ${ }^{27}$ As described in our report above, the neonate in case 2 experienced only minor respiratory morbidity after birth, leading us to believe that expectant management of a periviable abdominal pregnancy with the diagnosis of anhydramnios or PPROM is an appropriate option.

Table 1 MRI estimation of placental volume in case 2

\begin{tabular}{|l|l|}
\hline Occasion & Estimated placental volume $\left(\mathrm{cm}^{3}\right)$ \\
\hline On admission & 516.8 \\
\hline Before surgery & 790 \\
\hline 6 mo postdelivery & 283.3 \\
\hline
\end{tabular}

Abbreviation: MRI, magnetic resonance imaging.

Note: There was an increase of placenta volume with continued expectant management and significant spontaneous reduction of placenta volume left in place 6 months after delivery.
Postoperative methotrexate administration has been recommended by some authors if the placenta was left in situ. Others have discouraged such practice, as rapid placental degradation leads to accumulation of necrotic tissues and invites bacterial infection. ${ }^{13,28}$ We did not use methotrexate postoperatively since its mechanism of action is to inhibit rapidly dividing cells, which is not the case with a mature placenta in advanced abdominal pregnancy with limited proliferative activity.

Our experiences with the above-reported cases suggest that advanced abdominal pregnancy can be managed expectantly, particularly in the periviable stage of gestational age. Close inpatient monitoring of maternal and fetal conditions, adequate imaging of fetal anatomy and placental implantation location, and a multidisciplinary approach that includes the patient's input are paramount in achieving the appropriate management. Judicious planned surgical intervention with an individualized risk-versus-benefit assessment regarding leaving or removing the placenta is essential to achieve favorable outcomes.

\section{References}

1 Farquhar CM. Ectopic pregnancy. Lancet 2005;366(9485):583-591

2 Karaer O, Ilkgül O, Oruç S. Primary omental pregnancy on the gastrocolic ligament. South Med J 2007;100(4):403-404

3 Atrash HK, Friede A, Hogue CJ. Abdominal pregnancy in the United States: frequency and maternal mortality. Obstet Gynecol 1987;69(3 Pt 1):333-337

4 Ekele BA, Ahmed Y, Nnadi D, Ishaku K. Abdominal pregnancy: ultrasound diagnosis aided by the balloon of a Foley catheter. Acta Obstet Gynecol Scand 2005;84(7):701-702

5 Ramphal S, Moodley J. Advanced extrauterine pregnancy. Curr Obstet Gynaecol 1998;8(2):90-95

6 De Muelenaere HM. Advanced abdominal pregnancy and preeclampsia: A case report and literature review. Obstetrics and Gynaecology Forum 2003;13(3):22-26

7 Dahab AA, Aburass R, Shawkat W, Babgi R, Essa O, Mujallid RH. Full-term extrauterine abdominal pregnancy: a case report. J Med Case Reports 2011;5:531

8 Opare-Addo HS, Deganus S. Advanced abdominal pregnancy: a study of 13 consecutive cases seen in 1993 and 1994 at Komfo Anokye Teaching Hospital, Kumasi, Ghana. Afr J Reprod Health 2000;4(1):28-39

9 Worley KC, Hnat MD, Cunningham FG. Advanced extrauterine pregnancy: diagnostic and therapeutic challenges. Am J Obstet Gynecol 2008;198(3):297.e1-297.e7

10 Huang K, Song L, Wang L, Gao Z, Meng Y, Lu Y. Advanced abdominal pregnancy: an increasingly challenging clinical concern for obstetricians. Int J Clin Exp Pathol 2014;7(9):5461-5472

11 Mpogoro F, Gumodoka B, Kihunrwa A, Massinde A. Managing a live advanced abdominal twin pregnancy. Ann Med Health Sci Res 2013;3(1):113-115

12 Ludwig M, Kaisi M, Bauer O, Diedrich K. The forgotten child-a case of heterotopic, intra-abdominal and intrauterine pregnancy carried to term. Hum Reprod 1999;14(5):1372-1374

13 Roberts RV, Dickinson JE, Leung Y, Charles AK. Advanced abdominal pregnancy: still an occurrence in modern medicine. Aust N Z J Obstet Gynaecol 2005;45(6):518-521

14 Cotter A, Izquierdo L, Heredia F. Abdominal pregnancy. Available at: https://www.sonoworld.com/fetus/page.aspx?id=1032. Accessed April 15, 2016

15 Stanley JH, Horger EO III, Fagan CJ, Andriole JG, Fleischer AC. Sonographic findings in abdominal pregnancy. AJR Am J Roentgenol 1986;147(5):1043-1046 
16 Bertrand G, Le Ray C, Simard-Emond L, Dubois J, Leduc L. Imaging in the management of abdominal pregnancy: a case report and review of the literature. J Obstet Gynaecol Can 2009;31(1):57-62

17 Varma R, Mascarenhas L, James D. Successful outcome of advanced abdominal pregnancy with exclusive omental insertion. Ultrasound Obstet Gynecol 2003;21(2):192-194

18 Brouard KJ, Howard BR, Dyer RA. Hepatic Pregnancy Suspected at Term and Successful Delivery of a Live Neonate With Placental Attachment to the Right Lobe of the Liver. Obstet Gynecol 2015; 126(1):207-210

19 Patel C, Feldman J, Ogedegbe C. Complicated abdominal pregnancy with placenta feeding off sacral plexus and subsequent multiple ectopic pregnancies during a 4-year follow-up: a case report. J Med Case Reports 2016;10:37

20 Cotter AM, Jacques EG, Izquierdo LA. Extended field of view sonography: a useful tool in the diagnosis and management of abdominal pregnancy. J Clin Ultrasound 2004;32(4):207-210

21 Tasnim N, Mahmud G. Advanced abdominal pregnancy-a diagnostic and management dilemma. J Coll Physicians Surg Pak 2005; 15(8):493-495
22 Sentilhes L, Ambroselli C, Kayem G, et al. Maternal outcome after conservative treatment of placenta accreta. Obstet Gynecol 2010; 115(3):526-534

23 Marcellin L, Ménard S, Lamau MC, et al. Conservative management of an advanced abdominal pregnancy at 22 weeks. AJP Rep 2014; 4(1):55-60

24 Fouelifack FY, Fouogue JT, Fouedjio JH, Sando Z. Viable abdominal pregnancy: a case report in Yaoundé (Cameroon). Pan Afr Med J 2014;18:181

25 Smrtka MP, Gunatilake R, Miller MJ, Heine RP, Brown HL. Improving the management of an advanced extrauterine pregnancy using pelvic arteriography in a hybrid operating suite. AJP Rep 2012;2(1):63-66

26 Practice bulletins No. 139: premature rupture of membranes. Obstet Gynecol 2013;122(4):918-930

27 Kohl T, Geipel A, Tchatcheva K, et al. Life-saving effects of fetal tracheal occlusion on pulmonary hypoplasia from preterm premature rupture of membranes. Obstet Gynecol 2009;113(2 Pt 2):480-483

28 Rahman MS, Al-Suleiman SA, Rahman J, Al-Sibai MH. Advanced abdominal pregnancy-observations in 10 cases. Obstet Gynecol 1982;59(3):366-372 